\title{
Uncertainty in phylogenetic tree estimates
}

\author{
Amy Willis \\ Department of Biostatistics, University of Washington \\ and \\ Rayna Bell \\ Smithsonian Institution, National Museum of Natural History
}

October 16, 2017

\begin{abstract}
Estimating phylogenetic trees is an important problem in evolutionary biology, environmental policy and medicine. Although trees are estimated, their uncertainties are generally discarded in statistical models for tree-valued data. Here we explicitly model the multivariate uncertainty of tree estimates. We consider both the cases where uncertainty information arises extrinsically (through covariate information) and intrinsically (through the tree estimates themselves). The latter case is applicable to any procedure for tree estimation, and thus has broad relevance to the entire field of phylogenetics. The importance of accounting for tree uncertainty in tree space is demonstrated in two case studies. In the first instance, differences between gene trees are small relative to their uncertainties, while in the second, the differences are relatively large. Our main goal is visualization of tree uncertainty, and we demonstrate advantages of our method with respect to reproducibility, speed and preservation of topological differences compared to visualization based on multidimensional scaling. The proposal highlights that phylogenetic trees are estimated in an extremely high-dimensional space, resulting in uncertainty information that cannot be discarded. Most importantly, it is a method that allows biologists to diagnose whether differences between gene trees are biologically meaningful, or due to uncertainty in estimation.
\end{abstract}

Keywords: evolutionary histories, visualization, standard errors, dimension reduction 


\section{Introduction}

Information about the shared evolutionary history of organisms is critical in many different contexts. A correct understanding of evolutionary relationships and the mechanisms by which lineages diverge informs conservation efforts (Marko et al., 2011), environmental policy (Carpenter et al., 2011), and medicine (González-Candelas et al., 2013). Consequently, the field of phylogenetics, which concerns the inference of evolutionary relationships between organisms, is an active and well-developed subdiscipline of biology.

Collections of phylogenetic trees are common in phylogenetic inference and typically consist of individual phylogenetic trees each constructed from different parts of the genome, or of a subset of phylogenetic trees sampled from the posterior distribution of a phylogenetic analysis. Effective graphical representations of trees can quickly indicate whether a collection of trees represent similar evolutionary histories; however, visualizing collections of trees is challenging because phylogenetic trees are multivariate, tree-valued data objects.

Almost all phylogenetic trees depicted in the modern biological literature convey uncertainty by reporting the proportion of tree samples (bootstrap or posterior) on which the branch is present. However, this approach treats each branch as independent, ignoring the multivariate nature of trees. Here we propose a procedure that accounts for dependencies in the trees' branches, and models their uncertainty multivariately. Our proposal uses the log map of Barden et al. (2016) to map trees from their metric space to Euclidean space, where we model a tree estimate $\hat{T}$ as a noisy realization of the true tree $T$. Once we estimate the model parameters, we can then employ Euclidean multivariate analysis techniques to reduce the dimension of the trees, and visualize them, along with their uncertainties.

While one motivation for this paper is to propose a new method for modeling and visualizing uncertainty in trees, another motivating factor concerns the treatment of trees by mathematicians working with tree space. The metric space of phylogenetic trees was developed to assist with tree comparisons, tree estimation and quantification of uncertainty in trees (Billera et al., 2001). While substantial progress has been made towards the first two of these goals (Feragen et al., 2012, Chakerian and Holmes, 2012; Benner et al., 2014; Dinh et al., 2016), incorporating tree uncertainty into statistical models (which we distinguish from recent advances in pure probability) has been relatively untreated in the literature. Trees in tree space are almost always viewed as point masses, which falsely suggests that they are known with certainty. While flexible tree estimation methods are available (Ronquist et al., 2012; Bouckaert et al. 2014; Mirarab et al., 2014), phylogenetic trees are nonetheless estimated, and information regarding estimation precision is generally available. This paper advocates for the inclusion of this precision when considering trees as objects in their metric space.

The paper begins by introducing phylogenetic trees along with a discussion of existing tree visualization methods, including multidimensional scaling (Section 2). The log map is then proposed as a visualization tool for trees and tree uncertainty (Section 3). This is followed by a discussion of some 
sources of uncertainty in phylogenetic trees, and some methods for incorporating univariate uncertainty into tree visualization and modeling using the log map (Section 4). Multivariate uncertainty is then introduced (Section 5), and the advantages of the procedure described here are contrasted with multidimensional scaling (Section 6). The paper is concluded with some limitations of the log map (Section 7) and some final remarks (Section 8). All scripts and data are available via github.

\section{Tree visualization}

Phylogenetic trees are edge-weighted trees (acyclic graphs with no nodes of degree 2). They represent the evolutionary relationships of a collection of organisms that are known to share an ancestor. Internal vertices (vertices/nodes with degree 3 or greater) represent the shared ancestor that existed at a point in history, and the leaves (also called tips; vertices with degree one) represent modern (or observable, eg. via the fossil record) organisms. The length of the branches (the edge-weights) represents the extent of divergence between nodes. In some contexts the graph will have a "root" representing the common ancestor. In these cases the graph will be directed and the root will have out-degree one.

The space of possible trees is enormous. To illustrate, consider that a tree with $m+2$ leaves has at most $m$ internal branches of positive length, and there are $\frac{(2 m+2) !}{2^{m+1}(m+1) !}$ possible tree topologies. By comparison, there are $2^{m}$ orthants in $\mathbb{R}^{m}$. Since each tree can be associated with a single orthant in $\mathbb{R}^{m}$ (that reflects its branch lengths), it can thus be argued that tree space is

$$
\frac{(2 m+2) !}{2^{m+1}(m+1) !} / 2^{m}=\frac{(2 m+2) \times(2 m+1) \times \ldots \times(m+2)}{2^{2 m+1}}
$$

times larger than Euclidean space; that is, exponentially larger. Thus the space of trees with 6 leaves is more than 50 times larger than $\mathbb{R}^{4}$, and the space of trees with 10 leaves is more than 130,000 times larger than $\mathbb{R}^{8}$. As a result, representing collections of trees, especially large collections of trees sharing a large leaf set, necessitate some compression of the trees' information.

The particular form of tree space compression that we advocate in this paper is called the log map. The log map is a function that maps from tree space to Euclidean space, and was first proposed by Barden et al. (2016). It captures both topology (branching order) and branch length information about a tree. Here we present the log map as a modeling tool for both trees and tree uncertainty, arguing for its advantages as a native coordinate system for trees, in reproducibility of tree analyses, and for its capacity to reflect multivariate uncertainty in tree estimates.

Models and algorithms for estimating phylogenetic trees abound in the literature (Ronquist et al. 2012; Bouckaert et al., 2014; Mirarab et al., 2014; Binet et al., 2016). In this section we do not consider the problem of tree estimation. Instead, we wish to visualize a collection of trees that share a leaf set of $m$ taxa. These trees may arise via different models for tree estimation, as samples from a posterior distribution for a given gene's phylogeny, or as estimates of the phylogeny based on different genes. We 
denote them as $\hat{T}_{1}, \ldots, \hat{T}_{n}$, permitting them to be either rooted or unrooted. We wish to visualize this collection.

\subsection{Multidimensional scaling}

A common method of visualizing collections of trees is multidimensional scaling (Hillis et al., 2005; Chakerian and Holmes, 2012; Kendall and Colijn, 2016). Multidimensional scaling (MDS), first proposed by Torgerson (1952), is a powerful technique for mapping a collection of objects to vectors in $\mathbb{R}^{k}$, where $k$ is usually chosen to be 2 or 3 . The only requirement is a distance (or dissimilarity measure) between the objects. MDS involves finding a matrix that minimizes a stress function, which encodes the difference between the distances under the map and the true distances. The minimising matrix, in $\mathbb{R}^{n \times k}$, can then be visualized as $n$ points in $\mathbb{R}^{k}$. A common choice of stress function is the Kruskal- 1 function (Kruskal, 1964; Hillis et al., 2005), with the resulting map given by

$$
\arg \min _{x \in \mathbb{R}^{n \times k}}\left(\sum_{i \neq j} D_{i j}-\left|x_{i}-x_{j}\right|^{2}\right)^{1 / 2},
$$

where $\left\{D_{i j}\right\}_{(i, j) \in\{1, \ldots, n\}^{2}}=d\left(\hat{T}_{i}, \hat{T}_{j}\right)$ is the $n \times n$ matrix of distances between the tree-valued estimates $\hat{T}_{i}, \hat{T}_{j}$.

A key advantage of MDS is that the distance can be chosen to best highlight the differences of importance between the trees in the collection. Hillis et al. (2005) proposed using the Robinson-Foulds (RF) distance (Robinson and Foulds, 1981) to view the topological differences between trees. Chakerian and Holmes (2012) discussed the advantages of using the BHV distance to incorporate both topological and branch length features, and Gori et al. (2016) used this approach to cluster genes by phylogeny. Kendall and Colijn (2016) recently proposed a method for comparing differences between the most recent common ancestors of the tips, and Huang et al. (2016) describe an efficient implementation of MDS for many different tree metrics. We will contrast the advantages of MDS with the advantages of our procedure in Section 6

\subsection{DensiTree}

The program DensiTree (Bouckaert, 2010; Bouckaert and Heled, 2014) is a popular program for viewing collections of trees, and integrates seamlessly with the most prominent Bayesian tree estimation programs. DensiTree overlays the trees transparently, thus darker regions of the image imply greater confidence. Furthermore, small numbers of alternative topologies with comparable levels of support are easily observed. This tool can also show other parameters that are used in coalescent-based phylogenetic tree estimation, such as population size, by indexing the widths of the branches to these parameters. DensiTree has the advantage that the graphical representation shows the collection of trees as trees, rather than mapping the trees to Euclidean space. It effectively illustrates both topological and branch length 
disagreements, but performs most effectively when only a small number of topologies conflict and the tree has a relatively small number of leaves. Large leaf sets, or many conflicting topologies, are difficult to distinguish by eye. The new procedure that we propose here can clearly show conflicting clusters of trees, and works well when there are many such clusters. It is at the expense, however, of visualizing the tree directly.

\subsection{Other tree visualization methods}

Other methods for comparing phylogenetic trees have been proposed in the literature, and we briefly mention some that are designed for comparing more than two trees (we will not address pairwise comparisons given that our interest is in comparing large collections of trees, nor will we discuss methods for viewing individual trees). Sundberg et al. (2009) argue that the space of resolved trees can be mapped to an $n$-torus, and then uses cartographic projections to reduce dimension. However, this loses key branch length information and non-resolved trees are not permitted. TreeScaper (Huang et al., 2016) implements various tools for analyzing collections of trees, including estimating intrinsic dimensionality, and community detection. A dimension reduction tool that removes selected branches to aid longitudinal analysis has recently been proposed (Zairis et al., 2016), though the authors' goal was not visualization. Heatmaps can be used to summarize the dissimilarity matrix of MDS (Puigbò et al., 2007). Treemaps, which partition a rectangle into panels representing each tree and then display the nodes in a space-filling manner, may be used to see hierarchical dissimilarities (Tu and Shen, 2007), though its scalability with the size of the collection is limited. Trees of trees, proposed by Nye (2008), assigns the topologies of the trees in the collection to a "meta-tree"'s leaf nodes, then uses neighbour-joining methods to cluster the nodes (trees) by topolgical similarity. Hess et al. (2014) append histograms of related parameter estimates to leaves to show variation in these parameters (eg. population size) across the clades, while Bremm et al. (2011) developed PhyloComp to enable targeted comparison of differences at recent or deep divergence times (note the connection to the motivation of the metric proposed by Kendall and Colijn (2016)). treespace (Jombart et al., 2017), implemented as an R package, is a modern tool approach to analyzing distributions of trees, which is known to be a difficult problem. Most of these methods are designed to highlight topological differences, and few scale well both visually and computationally.

\section{The log map}

Each of the methods described above have distinct advantages in different situations. However, none have the ability to illustrate uncertainties in the $\left\{\hat{T}_{i}\right\}_{i}$ 's. The method that we propose here, which deals with this issue, utilizes a map from tree space to Euclidean space, called the log map. We review the construction of the log map here.

Consider the trees $T_{1}, \ldots, T_{n}$ with the same leaf set as objects in $\mathcal{T}_{m+3}$, the metric space of phyloge- 
netic trees (Billera et al. 2001). While tree space has many locally Euclidean properties, tree topologies are globally folded over each other, and thus visualizing the space directly is extremely difficult. Furthermore, because tree space is not equipped with an inner product, we have no concept of orthogonality or rotations on the space, which prohibited descriptions of covariance and central limit theorems until recently. To deal with the latter, Barden et al. (2016) developed the log map, $\Phi_{T^{*}}(T): \mathcal{T}_{m+3} \rightarrow \mathbb{R}^{m}$, which gives the Euclidean coordinates of a target tree $T$ relative to a base tree $T^{*}$. The following 4 cases are helpful in understanding the log map:

1. $\Phi_{T^{*}}\left(T^{*}\right)$ : The log map returns an arbitrary ordering of the $m$ branch lengths of $T^{*}$ (some of which may be zero for an unresolved tree $T^{*}$ ).

2. $\Phi_{T^{*}}(T)$ for $T$ with the same topology as $T^{*}$ : The log map returns the branch lengths of $T$ in accordance with the ordering of $\Phi_{T^{*}}\left(T^{*}\right)$.

3. $\Phi_{T^{*}}(T)$ for $T$ with a topology that is a single nearest neighbor interchange (NNI) from $T^{*}$ : The same as for Case 2 for all shared edges present on both trees, and the coordinate of the branch that is not shared is $-e_{T}$, where $e_{T}$ is the length of the branch present on $T$ but not on $T^{*}$.

4. $\Phi_{T^{*}}(T)$ for $T$ with a topology that is two or more NNIs from $T^{*}$ : Let $\gamma\left(T^{*}, T\right)$ be the geodesic distance between $T^{*}$ and $T$. Then $\Phi_{T^{*}}(T)$ is found by continuing for distance $\gamma\left(T^{*}, T\right)$ along a unit vector in the direction from $T^{*}$ towards the first bend in the BHV geodesic. See Willis (to appear) or Barden et al. (2016) for a more formal description.

We refer the reader to Barden et al. (2016) for a discussion of the case where $T^{*}$ falls on codimensional planes in $\mathcal{T}_{m+3}$.

In many ways, the log map is an ideal map because it preserves both local directions and BHV distances between trees (with respect to the base tree $T^{*}$ ). Some information must be discarded in any mapping from tree space to Euclidean space because tree space cannot be completely embedded in Euclidean space (e.g., Nye (2011, p. 2720)). However, while the topological information of trees is lost, the information as to whether or not a tree was one or more NNIs from the base tree is maintained: one NNI is given by a single negative coordinate, more than one NNI is denoted by multiple negative coordinates. We return to a discussion of this in Section 6 and Figure 5. The log map fulfills the suggestion of Holmes (2005) to map trees to $\mathbb{R}^{d}$, though the construction is very different to that of MDS.

\section{Modeling tree uncertainty using extrinsic information}

The log map preserves information with respect to both tree topology and branch lengths, which suggests its potential utility for both modeling and visualizing multivariate tree uncertainty. In this section we consider a model that incorporates covariate information about the precision in the tree estimates, 
which we call extrinsic information because it is not tree-valued. We first describe the model and then demonstrate it on an example.

\subsection{Model setup}

Consider trees $\hat{T}_{1}, \ldots, \hat{T}_{n}: n$ estimates of an underlying evolutionary history $T$. Suppose extrinsic, that is, non-tree, information is available regarding the precision of the estimates. The example that we will consider is information regarding the evolutionary rate of each of the genes whose gene trees are estimated by the $\hat{T}_{i}$ 's. However, the Fréchet variance (Benner et al., 2014; Benner and Bačák, 2014), a parsimony or likelihood score (Montealegre and St John, 2002), or a measure of the extent of taxon sampling could also be used. The setting is that each estimate $\hat{T}_{i}$ is associated with a known covariate $r_{i}$, which is proportional to the variance of the estimate in a manner we now define.

We begin by projecting the estimates to Euclidean space using the log map. The log map requires a base tree on which to ground the projection, and here we use the proposal of Willis (to appear) to ground the log map at the sample Fréchet mean of the estimates. However, we are now in the heteroscedastic case, and need to modify this accordingly. Thus we define our base tree as the weighted Fréchet mean

$$
\bar{T}:=\arg \min _{t \in \mathcal{T}_{m}} \sum_{i=1}^{n} \frac{1}{r_{i}} \gamma\left(\hat{T}_{i}, t\right)^{2},
$$

where $\gamma\left(t_{1}, t_{2}\right)$ is the distance between trees $t_{1}$ and $t_{2}$ in the BHV metric. Note that the CAT( 0$)$ property of the BHV space guarantees tree mean uniqueness (Bačák, 2014, Theorem 2.4), which is an additional reason for choosing it here.

We now suppose that the log maps of the estimates are independent, noisy realisations of the log map of the true tree, that is,

$$
\Phi_{\bar{T}}\left(\hat{T}_{i}\right)=\Phi_{T}(T)+\mathcal{N}\left(0, \sigma^{2} r_{i} I_{m}\right)
$$

where $T$ is the population analogue of $\bar{T}$ :

$$
T:=\arg \min _{t \in \mathcal{T}_{m}} \int_{\mathcal{T}_{P}} \frac{\gamma(T, t)^{2}}{r_{T}} d F(T),
$$

for $F$ the tree-generating process (e.g. the population dynamics process that drives speciation across the population of genes). This model is underpinned by the belief that all estimates reflect the underlying phylogenetic tree, but that their precision differs in accordance with the extrinsic information known about the tree. Both the log map of $T$ and the common variance parameter $\sigma^{2}$ can then be estimated using maximum likelihood:

$$
\begin{aligned}
\widehat{\Phi_{T}(T)} & =\frac{\sum_{i} \Phi_{\bar{T}}\left(\hat{T}_{i}\right) / r_{i}}{\sum_{i} 1 / r_{i}} \\
\hat{\sigma}^{2} & =\frac{1}{n} \sum_{i} \frac{\left(\Phi_{\bar{T}}\left(\hat{T}_{i}\right)-\widehat{\Phi_{T}(T)}\right)^{2}}{r_{i}} .
\end{aligned}
$$


We are now able to associate the estimates with their information content visually. Consider a set with measure $(1-\alpha)$ under a normal distribution with mean $\Phi_{\bar{T}}\left(\hat{T}_{i}\right)$ and variance $\hat{\sigma}^{2} r_{i} I_{m}$ (we suggest the minimum volume set, which is centred at $\Phi_{\bar{T}}\left(\hat{T}_{i}\right)$ ). This set exists in $\mathbb{R}^{m}$, and thus to assist visualization we suggest projecting it onto the span of the first two principal components of the $\left\{\hat{T}_{i}\right\}$ and visualizing it on this subspace. Note that these sets are not confidence nor prediction sets, but are useful analogues for viewing the variability in the tree estimates.

Invariably objections could be raised as to the choice of the noise model, which we modelled as a spherical multivariate normal distribution. Because we only have a single realisation of each tree, for identifiability our covariance matrices must be low rank. We consider using multiple tree estimates in Section 5 to relax this constraint. However, Gaussian distributional cross-sections may not be plausible (though note that no literature regarding the distribution of log map estimates has yet been developed). If a practitioner wished to relax the assumption of normality, the only necessary changes to the procedure would be recalculating the maximum likelihood estimates of Equations (5) and (6), and changing the contours of the visualization set. While model misspecification should be investigated and corrected as just described, the purpose of this procedure is visualization, not inference, and we argue that this objective can be achieved despite mild deviations from normality. See Feragen et al. (2012) and Amenta et al. (2015) for two-sample permutation-based tests for equality of tree means and variances, and Holmes (2005) for a more general discussion of tree testing.

We now demonstrate this approach in a situation where the evolutionary rate of genes is available. We conclude the discussion of extrinsic tree uncertainty modeling by noting that if multivariate information regarding tree uncertainty was available $\left(\left\{r_{i}^{(1)}, \ldots, r_{i}^{(\ell)}\right\}_{i}\right)$, it could be incorporated into the above procedure by modeling the perturbations by $\mathcal{N}\left(0, \sigma_{(1)}^{2} r_{i}^{(1)}+\ldots+\sigma_{(\ell)}^{2} r_{i}^{(\ell)}\right)$, subject to identifiability constraints (no linear dependence amongst the vectors $\left(\left\{r_{i}^{(1)}, \ldots, r_{i}^{(\ell)}\right\}_{i}\right)$.

\subsection{Evolutionary rate and phylogenetic uncertainty}

Comparing sets of phylogenetic trees, each estimated using different regions of the genome (e.g. loci), is complicated by variation in the evolutionary rates of those regions. Loci that evolve slowly contain few informative sites for estimating recent divergence events, while loci that evolve more quickly often contain more natural variation, or mutational saturation, than phylogenetic signal for resolving older divergence events (Wägele and Mayer, 2007; Baeza and Fuentes, 2013). The informativeness of a particular locus for resolving a particular tree (i.e. ancient versus recent divergence) is thus related to its evolutionary rate.

To illustrate how the visualization method described in Section 4.1 can be used to incorporate uncertainty from covariate information, we consider the relative evolutionary rate of 574 different genes shared by 42 mammals. The OrthoMam database (Ranwez et al., 2007; Douzery et al., 2014) contains estimates of the gene trees of these genes, which we call $\hat{T}_{1}, \ldots, \hat{T}_{574}$ (estimation details available in Ranwez et al. 

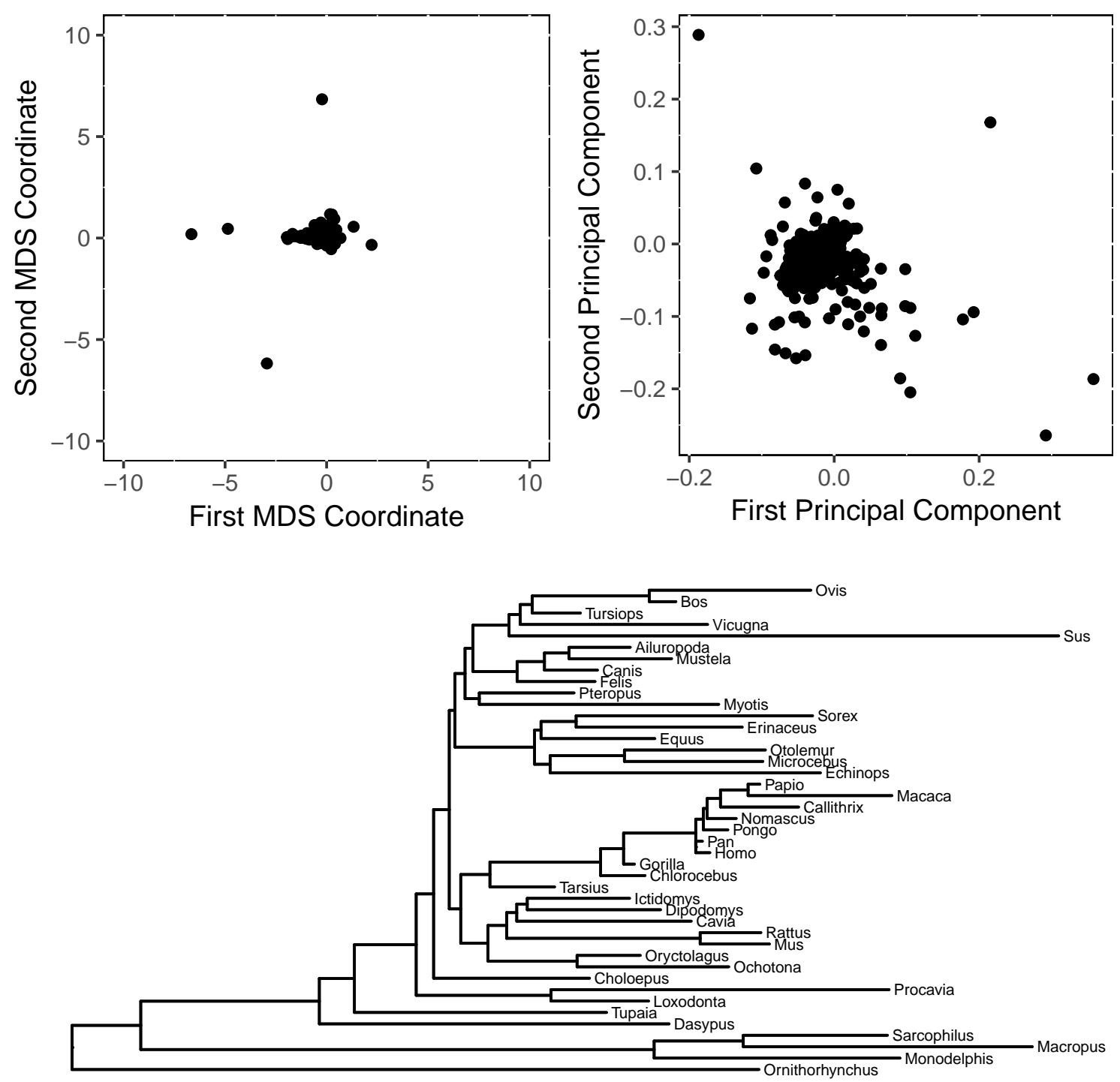

Figure 1: 574 gene trees shared by 42 mammals (Douzery et al., 2014). (top left) Multidimensional scaling of the BHV distances between the trees. (top right) The first two principal components of the log map of the trees with respect to their weighted Fréchet mean. Note that both representations suggest that the trees are known rather than estimated. (bottom) The SLC7A2 gene tree, one of the 574 gene trees mapped by the top figures.

(2007); the most commonly-selected model of best fit was GTR $+\Gamma$ ), along with the rates, which we call $r_{1}, \ldots, r_{574}$. Rate estimation was performed by Ranwez et al. (2007) via the Super Distance Matrix procedure of Adkins et al. (2001), which involves finding the rescaling of branches necessary to equalize leaf-to-tip distances.

Multidimensional scaling of the trees with respect to the BHV distance (Chakerian and Holmes, 2012) is shown in Figure 1 (top left), along with the first two principal components of the log maps of the trees (top right). The first 2 principal components explain $64 \%$ of the variation in the 40 -dimensional log mapped trees (see Supplementary Data for projections onto the third and fourth principal components). Both representations suggest that there are 4 trees that differ from the rest. MDS emphasizes this more heavily, most likely because the objective of MDS is to best capture all of the pairwise distances in the 

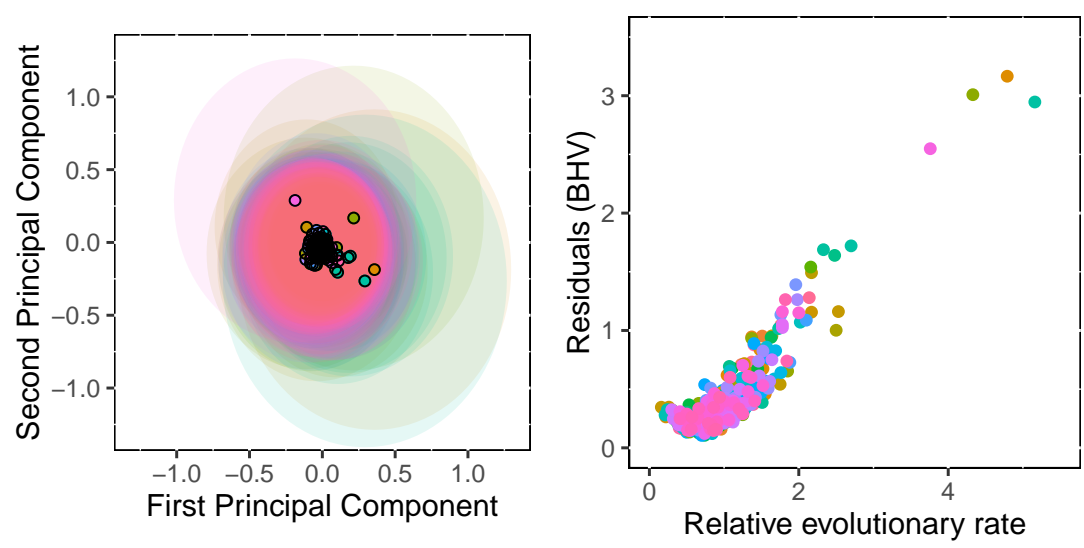

Figure 2: To account for estimation error, we construct a model for tree uncertainty (Section 4.1). (left) The 40-dimensional sets of volume 0.95 representing each tree, projected onto the first two principal components of the log maps of the tree estimates. Note that the sets appear large because they were constructed in a much higher dimensional space, and not because of the 4 "outliers" (see text). (right) The BHV distance from each gene tree to the weighted Fréchet mean shown against the relative evolutionary rates of the genes. Lower evolutionary rates, but not the lowest, correspond to the minimum distance trees.

mapping, while the log map instead preserves distances to the base tree $\bar{T}$. These 4 trees are topologically distinct from $\bar{T}$ by multiple nearest neighbor interchanges (see Supplementary Data).

We begin by calculating the weighted Fréchet mean of the gene trees $\bar{T}$ with weights $1 / r_{i}$ (Algorithm 4.2 of Bačák (2014) setting $\lambda_{k}=1 / k$; see also Miller et al. (2015)), and finding the log maps of the gene trees with respect to $\bar{T}, \Phi_{\bar{T}}\left(\hat{T}_{i}\right)$. We then calculate the maximum likelihood estimates of the model parameters (Equations $(5)$ and $(6)$ ), and construct 0.95 -measure sets with respect to a $\mathcal{N}\left(\Phi_{\bar{T}}\left(\hat{T}_{i}\right), \hat{\sigma}^{2} r_{i} I_{40}\right)$ distribution to illustrate the disagreement between the trees after accounting for the variance model. Because these sets are in $\mathbb{R}^{40}$, we project them onto the first two principal components 1 of the $\left\{\Phi_{\bar{T}}\left(\hat{T}_{i}\right)\right\}_{i}$. These projected sets are shown in Figure 2(left).

The impression given by Figure 1 suggests that there are a small number of trees that are very different from the rest of the collection. However, Figure 2 (left) makes it clear that relative to the uncertainty in the tree estimates, these trees are not especially outlying. A natural question to ask is to what extent the "outliers" inflate the estimate of $\sigma^{2}$, and removing these 4 points and recalculating the variance reduces it by only $5.4 \%$. Alas, the variance of the collection is only one component of the size of the sets; the other is the dimension. These log maps have been estimated in a 40-dimensional space, and the radius of these sets grows with the square root of the dimension. We argue that the high dimensionality of tree estimation leads to the uncertainty in estimation that we observe in Figure 2 (left). Note that while the

\footnotetext{
${ }^{1}$ Note that constructing the sets and then projecting them is computationally wasteful. For this reason we only determine the algebraic form of the $\mathbb{R}^{40}$ sets, and determine the form of the $\mathbb{R}^{2}$ sets algebraically before constructing them. Details are given as Supplementary Materials.
} 
sets in $\mathbb{R}^{40}$ sets are spherical, the rotation that we use is not, and for this reason the sets do not appear isotropic.

It is interesting to note the relationship between the distance between $\bar{T}$ and the $\Phi_{\bar{T}}\left(\hat{T}_{i}\right)$ 's and the $r_{i}$. While the form of Equation (2) places the greatest weight on gene trees with low evolutionary rate, we see from Figure 2 (right) that these are not the closest gene trees to the weighted average tree: trees corresponding to genes with small but non-minimal evolutionary rates are the minimum distance trees. We thus conjecture that the weighted Fréchet mean may provide a good estimate of the overall evolutionary process by incorporating the evolutionary rate information into tree estimation.

\section{Intrinsic uncertainty information}

Having discussed the case where covariate information is available regarding the tree estimates, we now consider the case where the trees themselves can be used for inferring the precision in estimation. We call this intrinsic information because it is tree-valued. In particular, suppose we wish to estimate $k$ different phylogenies $T^{(1)}, \ldots, T^{(k)}$. After describing the model in Section 5.1, we consider the case where these are the phylogenies of different genes in Section 5.2 .

\subsection{Intrinsic model setup}

The intrinsic uncertainty information comes from having $n_{i}$ estimates of $T^{(i)}$, which we call $\hat{T}_{1}^{(i)}, \ldots, \hat{T}_{n_{i}}^{(i)}$. Clearly this collection (which would most commonly arise from bootstrapping or posterior sampling) contains multivariate information about tree uncertainty: branches that can be estimated precisely would be present on all trees and with low variance in their lengths, while contentious branches may only appear on some trees or have huge variation in their lengths relative to the mean length. We wish to visualize these estimates and their multivariate uncertainty.

Define $\bar{T}^{(i)}$ to be the unweighted Fréchet mean of the $\left\{\hat{T}_{j}^{(i)}\right\}_{j}$ 's, $\bar{T}$ to be the unweighted Fréchet mean of the $\bar{T}^{(i)}$, and $T$ to be the population analogue of $\bar{T}$. We note that the latter may not have any biological significance, but we construct it order to have a common base for the log map. We now consider the model

$$
\Phi_{\bar{T}}\left(\hat{T}_{j}^{(i)}\right)=\Phi_{T}\left(T^{(i)}\right)+\mathcal{N}\left(0, \Sigma_{i}\right)
$$

The key difference between this model and that of Section 4.1 is that the uncertainty of the estimates is not constrained to be spherical: we permit $\Sigma_{i}$ to be unstructured. However, we can use the collection $\left\{\Phi_{\bar{T}}\left(\hat{T}_{j}^{(i)}\right)\right\}_{j}$ to estimate it. We suggest estimation via maximum likelihood if $n_{i}$ is large relative to the dimension of tree space, or a structured estimator if not.

Similar to the proposal of Section 4.1, we construct $(1-\alpha)$-volume sets of the distribution of the $\Phi_{\bar{T}}\left(\hat{T}_{j}^{(i)}\right)$, and again suggest the minimum volume sets. Again the sets will be $m$-dimensional, and 
we suggest projecting them to the subspace in $\mathbb{R}^{2}$ that spans the first two principal components of the $\left\{\Phi_{\bar{T}}\left(\hat{T}_{j}^{(i)}\right)\right\}_{i, j}$.

A key element of this visualization strategy is that it maintains $m$-dimensional uncertainty of tree estimation. Constructing a set based on a model for the principal components gives the impression of substantially more precision than truly exists, and MDS prohibits meaningful model constructions because the coordinate system is sample-dependent (discussed in Section 6). Visualizing uncertainty in tree estimates is an extremely important issue because of documented overconfidence in phylogeny estimates. Strong support for conflicting topologies can arise among phylogenies constructed from different sets of loci (e.g. sequence capture versus restriction site associated DNA sequencing [RADseq] (Leaché et al. 2015)) or from the same dataset analyzed under different models (e.g. concatenation versus multispecies coalescent (Edwards et al., 2016)). We hope our procedure assists with visualizing the uncertainty of phylogenetic estimates under these different scenarios.

\subsection{Multivariate tree uncertainty}

Here we will consider using samples from a posterior distribution on tree space to generate collections of tree estimates. However, the procedure proposed in Section 5.1 is agnostic with respect to the origin of the estimates ${ }^{2}$ Bootstrap resampling is another plausible method for generating collections of tree estimates.

Bayesian methods for estimating phylogenies naturally give rise to collections of trees as samples from the posterior. While sophisticated methods for summarizing samples from a posterior distribution on tree space exist (Section 2), tools to visualize the information that is lost in summarizing the collection of trees by a single tree are relatively underdeveloped.

To illustrate the advantages of the proposal of Section 5.1 to utilize collections of tree estimates to capture the multivariate tree uncertainty, we consider visualizing discordance between mitochondrial and nuclear gene phylogenies. In general, phylogenetic inferences based on different genes for a given set of taxa may differ with respect to topology and/or relative branch length due to poor gene tree reconstruction (e.g. due to mutational saturation and homoplasy) or because the gene trees differ from the underlying species tree (e.g. due to incomplete lineage sorting or introgression (Maddison, 1997; Rosenberg and Nordborg, 2002)). In particular, mitochondrial DNA can be especially problematic for resolving phylogenetic relationships at deeper evolutionary timescales due to its higher evolutionary rate of change. A reasonable visualization procedure should showcase the relative uncertainties in inferring the phylogenies of different loci.

\footnotetext{
${ }^{2}$ Substituting posterior standard deviations for frequentist standard errors overstates the precision in the estimates when priors are chosen to be uninformative (Efron et al., 1996; Efron, 2015), as is often the case in phylogenetic inference. The recent proposal of Efron (2015) could be applied to tree space in order to correct for this. However, because we are focusing only on an exploratory method, we defer the generalization of Efron's proposal to tree space to a separate investigation.
} 
Wiens et al. (2010) and Spinks and Shaffer (2009) investigated discordance between mitochondrial and nuclear loci in reconstructing evolutionary relationships among species of Emydid turtles. The large number of splits on the resulting tree estimates challenges fast identification of the differences between the mtDNA and nuDNA phylogeny estimates (see Wiens et al. (2010, Figures 1 and 2)). We selected a subset of 10 species that form a monophyletic group in the combined mtDNA and nuDNA analysis in Wiens et al. (2010) and combined data from Wiens et al. (2010), Spinks and Shaffer (2009) and Angielczyk et al. (2011) to create a complete data matrix for 1 mitochondrial and 9 nuclear loci. Sequences were aligned using Clustal X v.2.0.10 (Larkin et al., 2007). We used PartitionFinder v.1.1.0 (Lanfear et al. 2012) to determine the best fit substitution models and partitioning schemes for each locus (See Supplementary Material, Table 1) and estimated individual gene trees using Bayesian phylogenetic analyses implemented in Beast v.1.8.0 (Drummond et al., 2012) with a strict molecular clock and a speciation yule-process tree prior. We obtained posterior distributions from one independent Markov chain Monte Carlo simulation, and assessed convergence with Tracer v.1.5 (Rambaut and Drummond, 2013). After initial burn in of 100,000 trees, we took 100 samples from each posterior sampled at intervals of 100,000 trees. In this way we generated 10 gene trees $\times 100$ tree estimates on the same 10 species: $\left\{\hat{T}_{j}^{(i)}\right\}_{\{i=1, \ldots, 10, j=1, \ldots, 100\}}$ where $i$ indexes over the 10 genes and $j$ indexes over the posterior trees. We choose this level of thinning in order to generate a manageable number of near-independent draws from the posterior.

As described in Section 5.1, we construct sets for the gene trees in $\mathbb{R}^{7}$, before projecting them onto first 2 principal components of the $\left\{\hat{T}_{j}^{(i)}\right\}_{i, j}$ (Figure 3). Each gene tree generates its own covariance in accordance with the collection of estimates corresponding to that gene, for this reason the sets are not spherical nor self-similar.

We indeed notice discordance between the 1 mitochondrial (cytochrome $b$ gene) and the 9 nuclear genes, but no strong disagreement between the nuclear gene phylogenies. Most importantly, the discordance between mitochondrial and nuclear loci is present even after accounting for the uncertainty in estimating the gene trees. It is possible that the uncertainty in estimating such a high-dimensional object (a tree in $\mathcal{T}_{10}$ ) could have swamped the apparent differences between the mitochondrial and nuclear phylogenies, as was the case in Section 4.2. We advocate for the statistical paradigm that a difference between groups is not large unless it is large relative to estimation error.

\section{Contrasting MDS with the log map}

While the two procedures proposed in this paper have the advantage of a probabilistic interpretation that lend themselves to visualization of high-dimensional uncertainty, multidimensional scaling also has its advantages. For example, our procedure enforces that the distance between each tree estimate and the average tree (Fréchet mean) is measured according to the BHV distance. In contrast, the choice 

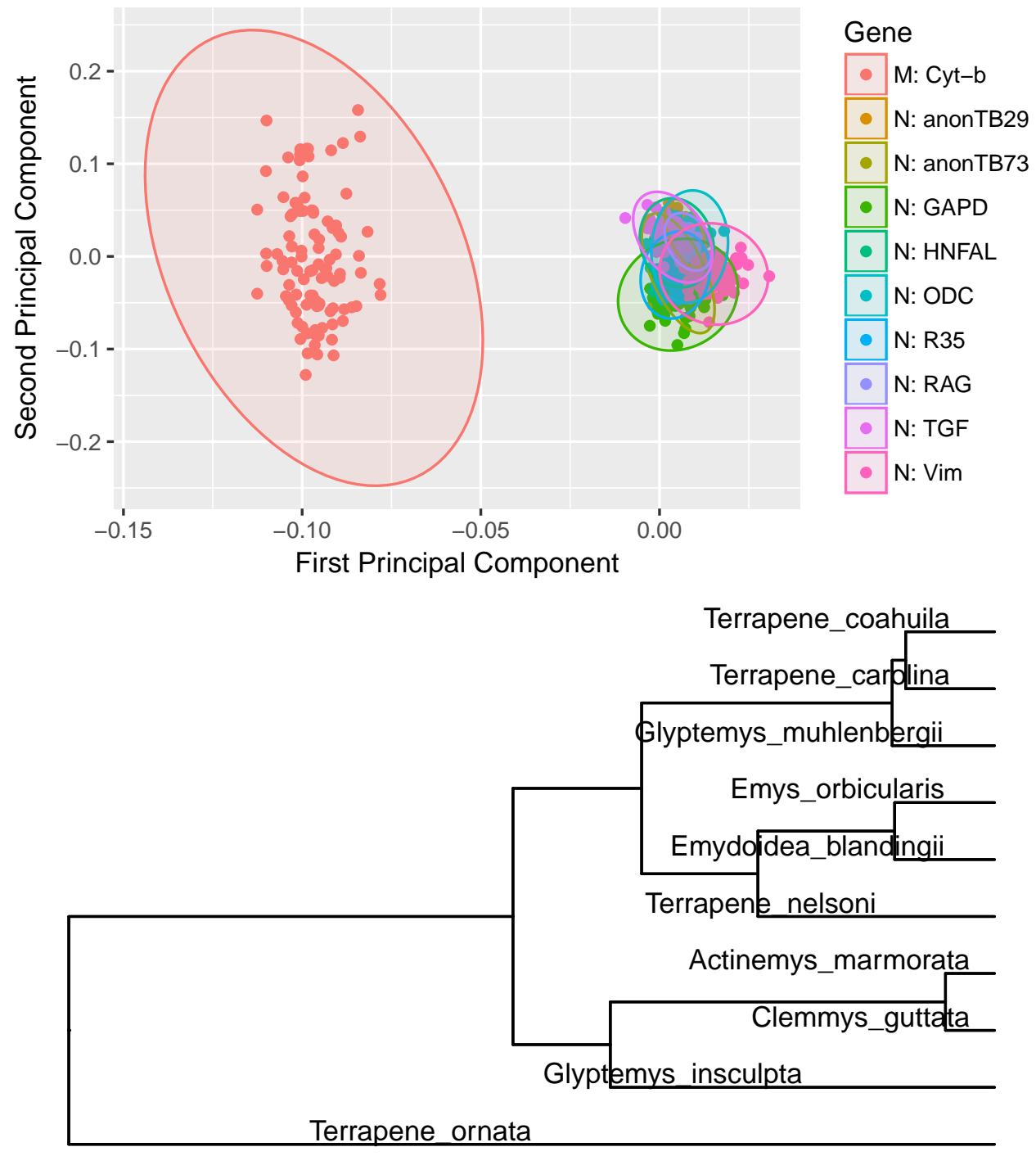

Figure 3: (top) The 7-dimensional sets reflecting the variability in estimating the phylogenies of 9 nuclear $(\mathrm{N})$ and 1 mitochondrial $(\mathrm{M})$ gene in 10 species of Emydid turtles. The projection onto the first two principal components, which explain $79 \%$ of the variance, of the estimates is shown (see Supplementary Data for projections onto the third and fourth principal components). We see that the difference between the nuclear and mitochondrial trees are large relative to the within-phylogeny estimation error. (bottom) One of the estimates of the Vim gene tree. 
of distance between trees in MDS can be selected to highlight the differing features between trees that are of greatest interest (Hillis et al., 2005; Chakerian and Holmes, 2012; Kendall and Colijn, 2016). In contexts where representing distances between trees is more important than displaying their estimation error, MDS will be a superior tool.

However, MDS has a number of serious drawbacks as a visualization method, some of which are not shared by the log map. We briefly describe five drawbacks of MDS that are improved by using the log map for visualization.

Visualizing uncertainty: MDS is a mapping rather than a rotation or projection, with the result that absolute measures of uncertainties in tree estimates cannot be preserved. In contrast, the same projection applied to the log maps can be applied to a set, and thus absolute sizes of uncertainties can be reflected (such as in Figures 2 and 3 .

Distortions surrounding equidistance: An ideal visualization procedure would communicate clearly which trees are equally distant from a central tree. Unfortunately, MDS fails to do this in many situations. To illustrate, we uniformly at random select a fully resolved tree topology with 50 leaves, and assign all branch lengths to unit length, and designate this tree as our "base tree." We then consider the collection of all trees that are one nearest neighbor interchange (NNI) from this tree. According to both the RF and BHV distance, all trees are distance 2 from the base tree. However, the projection to 3 dimensions by MDS using the Kruskal-1 stress function (Eqn. (1)) would suggest that some trees are substantially closer than others (Figure 4, left, modified from Hillis et al. (2005, Figure 10)). This can be very misleading when trying to identify representative trees or outliers. The log map, by construction, preserves distances to the base tree (Figure 4, right), though the compression of multiple topologies onto a single point is sacrificed.

Reproducibility: The stress-minimizing vector in MDS depends on every tree in the sample. As a result, a single new tree added to the sample may completely change the projection of all other points. Furthermore, visualizations cannot be compared across different studies, making the method inherently unreproducible. As reproducibility becomes an increasing focus of genetic studies, the importance of reproducible figures and visualization-based results is no less than reproducible quantitative analyses. As long as the base tree $\bar{T}$ and the PCA rotation matrices are maintained, the results of a new study can be compared alongside those of an original study ex post facto.

Topology versus branch length information: Negative coordinates in a log map indicate that the topology of the tree is different to that of the base tree. A single negative coordinate in a log map indicates that the target tree is a nearest neighbor interchange from the base tree. Thus the log map is capable of distinguishing topological versus branch length differences. This information is lost under MDS (on nonRF distances). Furthermore, if there is particular interest in a certain branch of the Fréchet mean tree, this coordinate could be plotted in order to investigate if a particular set of models or genes characterize the 


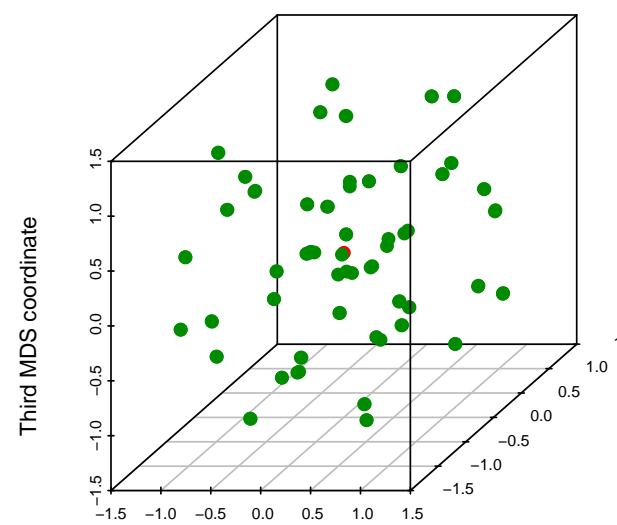

First MDS coordinate

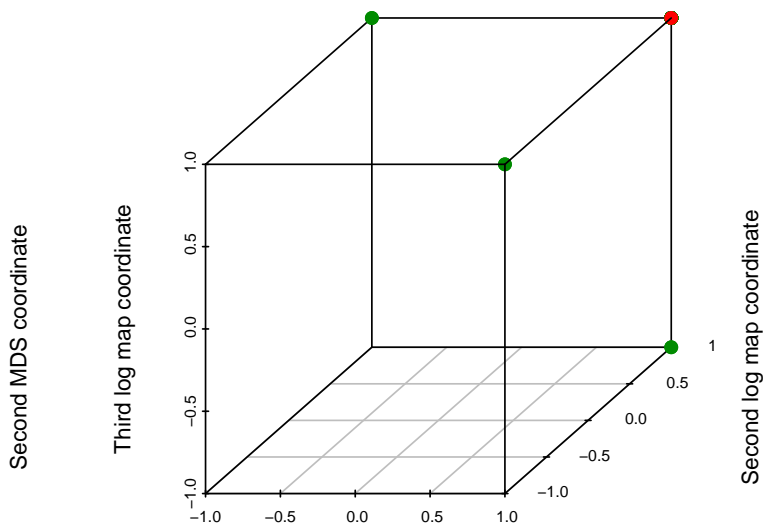

First log map coordinate

Figure 4: Multidimensional scaling may distort visualization of equidistant trees. Visualization of NNI trees (green) from a 50-taxon tree (red) under MDS on BHV distances using the Kruskal-1 stress function (left) and the log map (right). All trees are equidistant from the base tree. MDS distorts this, but the log map compresses trees onto one another.

presence of the branch. In Figure 5, we show two coordinates (branches) of the log map projection for the OrthoMam trees. The x-coordinate indicates the length of the branch separating the platypus from other marsupials (supported by $92 \%$ of trees), while the y-coordinate indicates the length of the branch separating the Human-Chimp-Gorilla clade from the remaining mammals (supported by $81 \%$ of trees). This information may be relevant to determining which clades on the mean tree are also supported by different genes.

Speed: Multidimensional scaling necessitates construction of the matrix of pairwise distances between trees, or calculation of $\frac{n \times(n-1)}{2}$ distances. This can be computationally prohibitive when distance calculations are expensive, as is the case for BHV distances. For example, in Section 4.2, constructing the BHV distance matrix for MDS required finding $\frac{574 \times 573}{2}=164,451$ geodesics. The procedure that we proposed required only 10,000 geodesics to update $\bar{T}$ until convergence, followed by 574 geodesics to calculate the $\left\{\Phi_{\bar{T}}\left(\hat{T}_{i}\right)\right\}_{i}$. In practice, using the implementation of the geodesic calculation by Nye (2011), this amounted to 16 hours of computation for MDS compared to 5 minutes for our log map procedure, because the geodesics calculated using Algorithm 4.2 become progressively shorter, reducing the computational intensity of successive geodesic calculations. Note that the more efficient mean calculation algorithms of Skwerer et al. (2015) could increase the relative computational gains of our method, while a dynamic geodesic algorithm (Skwerer and Provan, 2015) could be used to reduce the computation time of MDS.

Nonetheless, we emphasize that the flexibility with respect to the metric distance of interest, as well as the preservation of relative distances between trees, maintain MDS as a valuable visualization tool, and we encourage its concurrent use even in situations where the model-based approach proposed here 


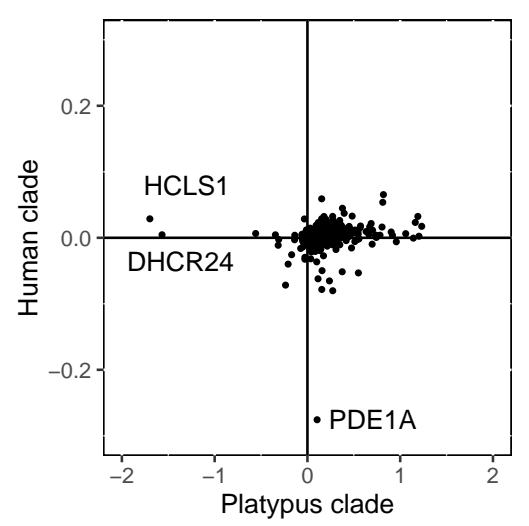

Figure 5: Two coordinates of the log-mapped OrthoMam gene trees. A negative coordinate in a log map indicates that the branch is absent on the tree, while a positive coordinate indicates its presence. In this way the log map can distinguish trees that are topologically distinct from trees that have only different branch lengths, which is not possible using MDS on BHV distances. In this figure, the $\mathrm{x}$-coordinate indicates the length of the branch separating the platypus from other marsupials, and the y-coordinate indicates the length of the branch separating the Human-Chimp-Gorilla clade from the remaining mammals. We give the names of three genes that are highly discordant on these branches.

may be useful.

\section{Limitations and open problems}

As discussed in Section 3, the log map does compress tree space, and it is not a bijection. Thus if a collection of trees was uniformly distributed throughout tree space, the log map may dissolve the most important information about the collection. However, in practice, collections of trees are rarely distributed uniformly throughout tree space, and a small number of topologies usually characterize most trees in the sample. For this reason, dimension reduction via the log map can preserve much of the structure present in the collection of trees. Indeed, this paper was motivated by attempts to find low rank approximations to tree collections. Nevertheless, unstructured tree datasets will be poorly reflected by the procedure proposed here.

Another criticism of the log map is that trees whose geodesic from the base tree follows the "cone path" will be mapped onto the line connecting the base tree to the star tree. However, only $23 \%$ of the Terrapene trees were a cone path from their mean tree, and zero of the 574 OrthoMam trees were a cone path from their mean tree. Furthermore, even if a large number of trees are cone path trees, this information can be used to determine which trees are highly topologically discordant. For example, 63\% of tree estimates for the ODC gene (Section 5) are cone path trees. Thus we argue that, contrary to distorting the space, the log map is a useful tool for quickly diagnosing extreme topological discordance 
in certain trees.

A curiosity of the models that were constructed in this paper is that they model the difference between tree estimates and true trees in Euclidean space, under the log map. It is more natural to model this difference in tree space, though unfortunately development of probability distributions on tree space is in its infancy. Recent work showing convergence of random walks to Brownian motion in tree space provide a promising avenue for construction of probability distributions on tree space (Nye, 2015). However, Brownian motion in tree space is (locally) spherical, and in its present form cannot describe non-spherical multivariate uncertainty in tree estimates. A promising direction of future research is the utility of probabilistic models in tree space for describing tree uncertainty, and comparisons with the models presented here.

A major limitation of almost all methods utilizing tree space is that missing leaves on some gene trees precludes their inclusion in the analysis. The procedure proposed here is no exception. While modern phylogenetic and phylogenomic data collection approaches (such as exon capture) are steadily reducing the amount of missing data, this remains a limitation.

\section{Concluding remarks}

We have proposed a method for incorporating tree-valued and non-tree-valued information into visualizations of trees and their uncertainty. Multidimensional scaling has many advantages for tree visualization, but because the representation only exists in the context of the sample, graphically incorporating the uncertainty that is lost in the map is not possible. By utilizing the log map to project the collection of trees to Euclidean space and modeling the projections as noisy realizations of the underlying evolutionary process, we may scrutinize differences between tree estimates alongside their variabilities. The result is an interpretable, exploratory method for observing discordance between gene trees, and among tree estimates. Further advantages of using PCA of log maps over multidimensional scaling include reproducibility and speed. We hope that the procedure and statistical perspective outlined in this paper reminds mathematicians working on tree space that trees are almost always estimated, and that differing levels of uncertainty needs to be accounted for when using tree space for analysis. However, most importantly, we hope to provide biologists with a method for diagnosing whether differences between gene trees are biologically meaningful, or due to uncertainty in estimation.

\section{Acknowledgements}

The authors are very grateful to an anonymous referee and two editors, whose helpful and constructive suggestions substantially improved both the text and figures of the manuscript. We are also grateful to the R Core Team (2017) and authors of the packages phangorn (Schliep, 2011), ape (Paradis et al., 2004), 
distory (Chakerian and Holmes, 2017), treespace (Jombart et al., 2017), MASS (Venables and Ripley, 2002), scatterplot3d (Ligges and Mächler, 2003), XML (Lang and the CRAN Team, 2017), gridExtra (Auguie, 2017), lattice (Sarkar, 2008), and ggplot2 (Wickham, 2009), which were used for constructing the figures and running the analyses in this paper.

\section{Supplementary Materials and Data}

All data and scripts utilized in this paper are available at https://github.com/adw96/TreeUncertainty, Details regarding model selection for Section 5 and projecting ellipses onto principal components are available in the same location.

\section{References}

Adkins, R. M., E. L. Gelke, D. Rowe, and R. L. Honeycutt (2001). Molecular phylogeny and divergence time estimates for major rodent groups: evidence from multiple genes. Molecular Biology and Evolution 18(5), 777-791.

Amenta, N., M. Datar, A. Dirksen, M. de Bruijne, A. Feragen, X. Ge, J. H. Pedersen, M. Howard, M. Owen, J. Petersen, J. Shi, and Q. Xu (2015). Quantification and visualization of variation in anatomical trees. In Research in Shape Modeling, pp. 57-79. Springer.

Angielczyk, K. D., C. R. Feldman, and G. R. Miller (2011). Adaptive evolution of plastron shape in Emydine turtles. Evolution 65(2), 377-394.

Auguie, B. (2017). gridExtra: Miscellaneous Functions for "Grid” Graphics. R package version 2.3.

Bačák, M. (2014). Computing medians and means in Hadamard spaces. SIAM Journal on Optimization 24(3), 1542-1566.

Baeza, J. A. and M. S. Fuentes (2013). Exploring phylogenetic informativeness and nuclear copies of mitochondrial DNA (numts) in three commonly used mitochondrial genes: mitochondrial phylogeny of peppermint, cleaner, and semi-terrestrial shrimps (Caridea: Lysmata, Exhippolysmata, and Merguia). Zoological Journal of the Linnean Society 168(4), 699-722.

Barden, D., H. Le, and M. Owen (2016, October). Limiting behaviour of Fréchet means in the space of phylogenetic trees. Annals of the Institute of Statistical Mathematics.

Benner, P. and M. Bačák (2014). Computing the posterior expectation of phylogenetic trees. ArXiv:1305.3692. 
Benner, P., M. Bačák, and P.-Y. Bourguignon (2014). Point estimates in phylogenetic reconstructions. Bioinformatics 30(17), 534-540.

Billera, L. J., S. P. Holmes, and K. Vogtmann (2001). Geometry of the space of phylogenetic trees. Advances in Applied Mathematics 27(4), 733-767.

Binet, M., O. Gascuel, C. Scornavacca, E. J. Douzery, and F. Pardi (2016). Fast and accurate branch lengths estimation for phylogenomic trees. BMC bioinformatics.

Bouckaert, R. and J. Heled (2014, December). DensiTree 2: Seeing Trees Through the Forest. bioRxiv.

Bouckaert, R., J. Heled, D. Kühnert, T. Vaughan, C.-H. Wu, D. Xie, M. A. Suchard, A. Rambaut, and A. J. Drummond (2014). BEAST 2: A Software Platform for Bayesian Evolutionary Analysis. PLoS Computational Biology 10(4), e1003537.

Bouckaert, R. R. (2010). DensiTree: making sense of sets of phylogenetic trees. Bioinformatics 26(10), 1372-1373.

Bremm, S., T. von Landesberger, M. Hess, T. Schreck, P. Weil, and K. Hamacherk (2011). Interactive visual comparison of multiple trees. In 2011 IEEE Conference on Visual Analytics Science and Technology (VAST), pp. 31-40. IEEE.

Carpenter, K. E., P. H. Barber, E. D. Crandall, M. C. A. Ablan-Lagman, Ambariyanto, G. N. Mahardika, B. M. Manjaji-Matsumoto, M. A. Juinio-Meñez, M. D. Santos, C. J. Starger, and A. H. A. Toha (2011). Comparative phylogeography of the Coral Triangle and implications for marine management. Journal of Marine Biology 2011(2), 1-14.

Chakerian, J. and S. Holmes (2012). Computational Tools for Evaluating Phylogenetic and Hierarchical Clustering Trees. Journal of Computational and Graphical Statistics 21(3), 581-599.

Chakerian, J. and S. Holmes (2017). distory: Distance Between Phylogenetic Histories. R package version 1.4.3.

Dinh, V., L. S. T. Ho, M. A. Suchard, and F. A. Matsen (2016). Consistency and convergence rate of phylogenetic inference via regularization. arXiv:1606.03059.

Douzery, E. J., C. Scornavacca, J. Romiguier, K. Belkhir, N. Galtier, F. Delsuc, and V. Ranwez (2014). OrthoMaM v8: A database of orthologous exons and coding sequences for comparative genomics in mammals. Molecular Biology and Evolution 31(7), 1923-1928.

Drummond, A. J., M. A. Suchard, D. Xie, and A. Rambaut (2012). Bayesian phylogenetics with BEAUti and the BEAST 1.7. Molecular Biology and Evolution 29(8), 1969-1973. 
Edwards, S. V., Z. Xi, A. Janke, B. C. Faircloth, J. E. McCormack, T. C. Glenn, B. Zhong, S. Wu, E. M. Lemmon, A. R. Lemmon, and others (2016). Implementing and testing the multispecies coalescent model: a valuable paradigm for phylogenomics. Molecular Phylogenetics and Evolution 94, 447-462.

Efron, B. (2015). Frequentist accuracy of Bayesian estimates. Journal of the Royal Statistical Society: Series B (Statistical Methodology) 77(3), 617-646.

Efron, B., E. Halloran, and S. Holmes (1996). Bootstrap confidence levels for phylogenetic trees. Proceedings of the National Academy of Sciences 93(23), 13429-13429.

Feragen, A., J. Petersen, M. Owen, P. Lo, L. H. Thomsen, M. M. Wille, A. Dirksen, and M. de Bruijne (2012). A hierarchical scheme for geodesic anatomical labeling of airway trees. In International Conference on Medical Image Computing and Computer-Assisted Intervention, pp. 147-155. Springer.

González-Candelas, F., M. A. Bracho, B. Wróbel, and A. Moya (2013). Molecular evolution in court: analysis of a large hepatitis $\mathrm{C}$ virus outbreak from an evolving source. BMC Biology.

Gori, K., T. Suchan, N. Alvarez, N. Goldman, and C. Dessimoz (2016). Clustering Genes of Common Evolutionary History. Molecular Biology and Evolution 33(6), 1590-1605.

Hess, M., S. Bremm, S. Weissgraeber, K. Hamacher, M. Goesele, J. Wiemeyer, and T. von Landesberger (2014). Visual Exploration of Parameter Influence on Phylogenetic Trees. IEEE Computer Graphics and Applications 34(2), 48-56.

Hillis, D. M., T. A. Heath, and K. St John (2005). Analysis and visualization of tree space. Systematic Biology 54(3), 471-482.

Holmes, S. (2005). Statistical approach to tests involving phylogenies. In Mathematics of Evolution and Phylogeny, pp. 91-120.

Huang, W., G. Zhou, M. Marchand, J. R. Ash, D. Morris, P. Van Dooren, J. M. Brown, K. A. Gallivan, and J. C. Wilgenbusch (2016). TreeScaper: Visualizing and Extracting Phylogenetic Signal from Sets of Trees. Molecular Biology and Evolution 33(12), 3314.

Jombart, T., M. Kendall, J. Almagro-Garcia, and C. Colijn (2017). treespace: exploration of landscapes of phylogenetic trees.

Kendall, M. and C. Colijn (2016, October). Mapping Phylogenetic Trees to Reveal Distinct Patterns of Evolution. Molecular Biology and Evolution 33(10), 2735-2743.

Kruskal, J. B. (1964). Multidimensional scaling by optimizing goodness of fit to a nonmetric hypothesis. Psychometrika 29(1), 1-27. 
Lanfear, R., B. Calcott, S. Y. W. Ho, and S. Guindon (2012). PartitionFinder: combined selection of partitioning schemes and substitution models for phylogenetic analyses . Molecular Biology and Evolution 29(6), 1695-1701.

Lang, D. T. and the CRAN Team (2017). XML: Tools for Parsing and Generating XML Within R and S-Plus. R package version 3.98-1.9.

Larkin, M. A., G. Blackshields, N. P. Brown, R. Chenna, P. A. McGettigan, H. McWilliam, F. Valentin, I. M. Wallace, A. Wilm, R. Lopez, J. D. Thompson, T. J. Gibson, and D. G. Higgins (2007). Clustal W and Clustal X version 2.0. Bioinformatics 23(21), 2947-2948.

Leaché, A. D., A. S. Chavez, L. N. Jones, J. A. Grummer, A. D. Gottscho, and C. W. Linkem (2015). Phylogenomics of phrynosomatid lizards: conflicting signals from sequence capture versus restriction site associated DNA sequencing. Genome biology and evolution 7(3), 706-719.

Ligges, U. and M. Mächler (2003). Scatterplot3d - an r package for visualizing multivariate data. Journal of Statistical Software 8(11), 1-20.

Maddison, W. P. (1997). Gene Trees in Species Trees. Systematic Biology 46(3), 523-536.

Marko, P. B., H. A. Nance, and K. D. Guynn (2011). Genetic detection of mislabeled fish from a certified sustainable fishery. Current Biology 21(16), 621-622.

Miller, E., M. Owen, and J. S. Provan (2015). Polyhedral computational geometry for averaging metric phylogenetic trees . Advances in Applied Mathematics 68, 51-91.

Mirarab, S., R. Reaz, M. S. Bayzid, T. Zimmermann, M. S. Swenson, and T. Warnow (2014, August). ASTRAL: genome-scale coalescence based species tree estimation. Bioinformatics, 1-8.

Montealegre, I. and K. St John (2002). Visualizing restricted landscapes of phylogenetic trees. In Proceedings of the European Conference for Computational Biology.

Nye, T. (2008). Trees of Trees: An Approach to Comparing Multiple Alternative Phylogenies. Systematic Biology 57(5), 785-794.

Nye, T. M. (2011). Principal components analysis in the space of phylogenetic trees. The Annals of Statistics 39(5), 2716-2739.

Nye, T. M. (2015). Convergence of random walks to Brownian motion in phylogenetic tree-space. arXiv:1508.02906.

Paradis, E., J. Claude, and K. Strimmer (2004). APE: analyses of phylogenetics and evolution in R language. Bioinformatics 20, 289-290. 
Puigbò, P., S. Garcia-Vallvé, and J. O. McInerney (2007). TOPD/FMTS: a new software to compare phylogenetic trees. Bioinformatics 23(12), 1556-1558.

R Core Team (2017). R: A Language and Environment for Statistical Computing. Vienna, Austria: R Foundation for Statistical Computing.

Rambaut, A. and A. Drummond (2013). Tracer 1.6. University of Edinburgh, Edinburgh, UK. Technical report.

Ranwez, V., F. Delsuc, S. Ranwez, K. Belkhir, M.-K. Tilak, and E. J. Douzery (2007). OrthoMaM: a database of orthologous genomic markers for placental mammal phylogenetics. BMC Evolutionary Biology 7(1), 1.

Robinson, D. F. and L. R. Foulds (1981). Comparison of phylogenetic trees. Mathematical Biosciences 53(1), 131-147.

Ronquist, F., M. Teslenko, P. van der Mark, D. L. Ayres, A. Darling, S. Höhna, B. Larget, L. Liu, M. A. Suchard, and J. P. Huelsenbeck (2012). MrBayes 3.2: Efficient Bayesian phylogenetic inference and model choice across a large model space. Systematic Biology 61(3), 539-542.

Rosenberg, N. A. and M. Nordborg (2002). Genealogical trees, coalescent theory and the analysis of genetic polymorphisms. Nature Reviews Genetics 3(5), 380-390.

Sarkar, D. (2008). Lattice: Multivariate Data Visualization with R. New York: Springer. ISBN 978-0387-75968-5.

Schliep, K. (2011). phangorn: phylogenetic analysis in r. Bioinformatics 27(4), 592-593.

Skwerer, S. and S. Provan (2015). Dynamic Geodesics in Treespace via Parametric Maximum Flow. arXiv:1512.03115.

Skwerer, S., S. Provan, and J. S. Marron (2015). Relative Optimality Conditions and Algorithms for Treespace Fréchet Means . arXiv:1605.02082.

Spinks, P. Q. and H. B. Shaffer (2009). Conflicting mitochondrial and nuclear phylogenies for the widely disjunct Emys (Testudines: Emydidae) species complex, and what they tell us about biogeography and hybridization. Systematic Biology 58, 1-20.

Sundberg, K., M. Clement, and Q. Snell (2009, September). Visualizing Phylogenetic Treespace Using Cartographic Projections. In Algorithms in Bioinformatics, pp. 321-332. Berlin, Heidelberg: Springer Berlin Heidelberg.

Torgerson, W. S. (1952). Multidimensional scaling: I. Theory and method. Psychometrika 17(4), 401419. 
Tu, Y. and H.-W. Shen (2007). Visualizing changes of hierarchical data using treemaps. IEEE Transactions on Visualization and Computer Graphics 13(6), 1286-1293.

Venables, W. N. and B. D. Ripley (2002). Modern Applied Statistics with S (Fourth ed.). New York: Springer. ISBN 0-387-95457-0.

Wägele, J. W. and C. Mayer (2007). Visualizing differences in phylogenetic information content of alignments and distinction of three classes of long-branch effects. BMC Evolutionary Biology 7(1), 1.

Wickham, H. (2009). ggplot2: Elegant Graphics for Data Analysis. Springer-Verlag New York.

Wiens, J. J., C. A. Kuczynski, and P. R. Stephens (2010). Discordant mitochondrial and nuclear gene phylogenies in Emydid turtles: implications for speciation and conservation. Biological Journal of the Linnean Society 99(2), 445-461.

Willis, A. (to appear). Confidence sets for phylogenetic trees. Journal of the American Statistical Association.

Zairis, S., H. Khiabanian, A. J. Blumberg, and R. Rabadan (2016). Genomic data analysis in tree spaces. arXiv:1607.07503. 